\title{
A Review on Flexible Spiral Wire Retainer
}

\author{
Dr. Mohamed Parshan K. A., Dr. Ravindra Kumar Jain, \\ Dept of Orthodontics, Saveetha University, India
}

\begin{abstract}
Maintaining newly moved teeth in position, long enough to aid in stabilizing their correction. Bonded retainers are type of retainers which does not require patient's compliance, invisiblity and long-term retention. This type of retainers also can produce rare undesirable tooth movement and effects on periodontium which is discussed in our review.
\end{abstract}

Keywords: Flexible spiral wire retainers, bonded retainers, multistranded wire retainers.

\section{Introduction}

Moyer'sdefined retention as 'maintaining newly moved teeth in position,long enough to aid in stabilizing their correction. Teeth that have been moved in or through bone by mechanical appliances have a tendency to return to their former position andthe purpose of retention is to counteract this tendency. Since retention requirements are uniquefor every casetheorthodontist has to opt for an appropriate appliance available to him. ${ }^{1}$ The various retentive appliances available include Bandedretainers, Removable retainers and bonded retainers. $^{2,3}$ Need for long term retention to avoid relapse is widely accepted now-a-days and hence bonded orthodontic retainers have found an important role in orthodontic treatment. Bonded orthodontic retainers consist of a length of orthodontic wire bonded to the teeth with acid-etch retained composite. Themajor advantages of bonded retainers overremovable retainers include, invisibility, no patient compliance required and long term retention.Bonded retainers used in the last decade include fixed rigid wire retainer and the Flexible spiral wire retainer . ${ }^{4}$ This review is focused mainly on the Flexible spiral wire retainers that are used now-a-days in orthodontics.

\section{Evolution Of Flexible Spiral Wire Retainers}

Since introduction of acid etch techniquebyBuonocore in 1955, Bonded retainers have become popular. In 1965, Newman ${ }^{5}$ first bonded orthodontic attachment to tooth and Kneirim ${ }^{6}$ published report of use of this technique to construct bonded retainers.Latermultistranded wire fixed retainers were constructed by Artun and Zachrisson $^{7}$ which were bonded from canine to canine.In 1983, Zachrisson ${ }^{8}$ reported useofmultistrandedwire bonded to all the teeth in labial segment. Many variations like different diameters, different composite, and use of mesh pads were also suggested ${ }^{9-10}$. In last 20 years Flexible spiral wire have evolved in which smalldiametermultistranded wire usually 0.0175 or 0.0215 inch is bonded to each tooth in the labial segment.

\section{Advantages Of Flexible Spiral Wire Retainersgiven By Graber}

1. Increase retention because of irregular surface ${ }^{8}$.

2. Invisible.

3. Neat and clean.

4. Can be used along with removable retainers.

5. They can be placed out of occlusion in most instances.

6 . Physiological movements of teeth are allowed by these retainers ${ }^{11}$.

\section{Disadvantages Of Flexible Spiral Wire Retainers:}

1. Good oral hygiene of the patient is mandatory.

2. Excessive adhesive during retainer bonding can cause gingival recession.

3. Undesirable movements can occur.

4.Not indicated in deep bite as they are under mechanical stress.

Indications Of Flexible Spiral Wire Retainers As Told By Zachrisson ${ }^{8}$ :

1. Closed midline diastema.

2. Anterior teeth spacing.

3. Potential post orthodontic tooth migration in adults.

4. Accidental loss of maxillary incisors, requiring closure, and retention of large anterior spaces.

5. Spacing reopening, after mandibular incisors extraction.

6.Palatally impacted canines. 


\section{Wire used:}

\section{Construction}

Stainless steel wire is mostly used. Multistranded wires may be round or rectangular formed by three to six fine strands braided or arranged co-axially. Diameter of wire varies from $0.015^{13-14}$ to 0.032 inch $^{7}$.

\section{Composite resin:}

Bonded fixed retainers are attached to the teeth with composite resin. Concise (3 M Unitekcorp.,Monrovia,calif.) a conventional restorative composite based on Bis-GMA. This is most commonly used.

Artun and Zachrisson ${ }^{7}$ diluted the concise restorative material using unfilled resinto improve handling properties for orthodontic bonding.

Other adhesives used are Sevritron ${ }^{6}$, Nuva-fil $^{15,16}$, Silux ${ }^{17}$, Bondmor ${ }^{18}$,Heliosit orthodontic ${ }^{19-20}$ and Transbond ${ }^{21-22}$.

\section{Direct technique:-}

\section{Techniques Of Fabrication}

Russel .D suggested the Length of wire to be fabricated is verified in latest cast. Loops are not required at end of the wire. Wire is again verified in patient's mouth clinically to ensure it fits passively to the tooth to be bonded and the tooth to be bonded were pumiced and etched. Wire is then accurately located on the teeth and bonded using composite resin. Indirect technique was suggested by Hobbson ${ }^{21}$.

\section{Long Term Effectiveness Of FSW}

According to 2 years study doneby Stormann ${ }^{23}$, norelapse was observed in patient with 0.0195 inch flexible spiral wirecanine to canine retainers, whereas 20\%relapse was observed in $0.0215 \mathrm{inch}$ FSW. However in both FSW groups the changes in irregularity index was not significant.

In recent study by Atack, Harradin, Sandy ${ }^{24} 0.065$ mean increase in irregularity index was shown 1 year after treatment with FSW. But it is notsignificantly different when compared to patients who received removable retainers.

\section{Survival Rate Of FSW}

According to Artun, failure rate with thick spiral wire bonded only to canine was $30.8 \%$ and thin spiral wire bonded to each tooth was $27.3 \%$.

Artun concluded that bonded orthodontic canine to canine retainers (plain and spiral wire) effectively maintain incisor alignment following orthodontic treatment however the failure rate of approximately $20 \%$ may be expected during a period of 3 years. Occasional cases of slight incisor relapse may occur using retainers bonded only to canines. ${ }^{25}$

According to study done by Lie Foeket al ${ }^{26}$ who used a flexible, braided, rectangular stainless steel wire retainer(Quad cat, GAC International).

1. The total survival rate of flexible, braided lingual retainerswas $63 \%$ over an observationperiod of 41.7 months and the highest number of failures was observed within the first 6 months after placement.

2. Failure rate of the mandible was $31.6 \%$ lower than the findings of and renetal who reported failure rate of $35 \%$.

3. Survival rate for mandible was $68.4 \%$.

4. Most failure occurred during first 6 months .Gender, age of the patient and operator experience did not affect the failure rate

According to Stormann and Ehmer $(2002)^{23}$, the failure rate for 0.0195 inch multistrandedwirefsw was $18 \%$ and for 0.0215 inch MS FSW was $53 \%$

Dahl and Zachrisson ${ }^{27}$ reported a failure rate of $10.3 \%$ with the use of 3 stranded spiral wire and $5.9 \%$ with the 5 stranded spiral wires. They concluded higher failure rate for maxilla compared to mandible. Excellent long-term success rate for six unit mandibular and four unit maxillary retainers was noted. Adequate plaque control by the patient is required and remaking retainers every 10 year once is recommended.

\section{Effects Of FSW In Periodontium}

N.Pandisetaldid a study on the periodontal status of long term mandibular lingual fixed retention. This study suggests long term retention promotes calculus accumulation,marginal recession, increased periodontal disease but has no effect on plaque and gingival index or bone level. ${ }^{28}$

But failure rates was considerably lower than those reported in other recent studies of lingual retainer over period of 20 years ${ }^{29-30}$.The results with 5 stranded penta-one wire are partiallyencouraging. 
According to Artun spiral wire retainer do not accumulate more plaque and calculus than the plain wire retainers and formation of caries is not a problem.the presence of bonded retainer did not seem to have any negative effect on the patient's ability to achieve satisfactory oral hygiene. Gingival health is significantly better at the 3 year follow up examination. ${ }^{25}$

\section{Unexpected Complications Of FSW}

From the study of Renkema et al ${ }^{31}$ it can be concluded that FSW canine to canine retainer is effective in maintaining the intercanine distance after orthodontic treatment, but in few patients unexpected complications has been reported like,torque difference between the adjacent mandibular incisors,increased inclination and movement of mandibular canine.Katsora ${ }^{32}$ observed same post treatment changes occurred in his 21 patients.

From the study made by Dahl and Zachrission ${ }^{27}$, Loosening and fracture of wire is less seen in $5 \mathrm{SW}$ than $3 \mathrm{SW}$, $62 \%$ of patient has evident abrasion of composite, $11 \%$ of patient had obvious wear facets in retainer wire, Opening of small spaces $(0.5-2 \mathrm{~mm})$ distal to terminal ends of intact retainer, Moderate gingivitis was observed in some patient due to inadequate hygiene. Accumulation of plaque along mandibular retainer was common .No signs of dental caries was seen.

\section{Conclusion}

In retention of the orthodontically treated teeth, Flexible spiral wire retainers does not requires patients compliance, they are invisible, aids in long term retention. In spite of some rare complications ,many orthodontists opt flexible spiral wire retainers as best.

\section{References}

[1] Zachrission .Excellence in finishing. Journal of Clinic Orthodontics 1986; 20; 460-82 and 536-56.

[2] Angle. Treatment of malocclusion of the teeth.7th edition.Philadelphia: SS White Manufacturing Co., 1907.

[3] Hawley CA. A Removable retainer.International Journal of Orthodontics. 1919; 2:291-8.

[4] David Russel .Bonded Orthodontic Retainer: A review. American Journal of Orthodontics and Dentofacial Orthopaedics.1995;108;207-13.

[5] Newman G.V. Epoxy adhesive for orthodontic attachments; Progress report. American journal of Orthodontics1965;51; 901-12.

[6] Kneirim RW .Invisible lower cuspid to cuspidretainer.Angle Orthodontics 1973; 43; 218-19.

[7] Artun.J,Zachrisson .Improving the handling properties of composite resin for direct bonding. American Journal of Orthodontics $1982 ; 81 ; 269-76$.

[8] Zachrisson. The bonded lingual retainer and multiple spacing of anterior teeth. Journal of Clinical Orthodontics 1983; 17;838-44.

[9] Chen RS. Prefabricated bonded mandibular retainer. Journal of Clinical Orthodontics 1978; 12; 788-9

[10] Weisser .Bonded twin lower 3-3 retaining arch. Journal of Clinical Orthodontics 1978; 12; 557-60.

[11] Artun.J .Caries and periodontal relation associated long term use of different types of bonded lingual retainers. American Journal of Orthodontics 1984; 86; 112-8.

[12] Saravana. Post orthodontic splinting of periodontally involved teeth. British Journal of Orthodontics 1990; 17; 29-32

[13] Sheridan J.J. Incremental removal of bonded lingual retainers. Journal of Clinical Orthodontics.

[14] Chan K.C, Andreasen .Conseravtive retention for maxillary central incisors. American Journal of Orthodontics $1975 ; 67$.

[15] Klassman,Zucker .Combination wire-composite intracoronal splinting, rationale and technique. American academy of periodontology.1976;47;481-6

[16] Paulson R.C.A Functional rationale for routine maxillary bonded retention.Angle Orthodontics; 1992; 62; 223-6.

[17] Lubit EC .The bonded lingual retainer. Journal of clinical Orthodontics.1979; 13 311-13

[18] Ferguson JW. Multistranded wire retainers; an indirect technique. British Journal of Orthodontics; $1987 ; 15 ; 51-4$

[19] Willmot DR, Davis . The use of light cure resin and orthodontic arch wire to immobilize a transplanted maxillary canine. British Journal of Orthodontics $1991 ; 170 ; 267-8$

[20] Hobson RS, Eastaugh DP. Silicon putty splint for rapid placement direct bonded retainers. Journal of Clincal Orthodontics.

[21] Hamula. Direct bonding with light cured adhesive. Journal of Clinical Orthodontics 1991; 25; 437-8.

[22] Stormann et al. Prospective randomized study of different retainers type. Journal of Orofacial and Orthopedics 2002; 63; $42-50$

[23] Atack et al, Which way forward? Fixed or Removable lower retainers.Angle orthodontics.2007; 77; 954-9.

[24] Artun, Agnes ,Peter : A 3- year follow up study of various types of orthodontic canine to canine retainers. European Journal of Orthodontics :19 (1997) :501-9

[25] D.J Lie Sam Foek et al, European Journal of Orthodontics 30 (2008) 199-204.

[26] Dahl and Zachrisson; Long term experience with direct bonded lingual retainers. Journal of Clinical Orthodontics.Oct 91 vol 25. Number 10: 619-630.

[27] N.Pandis et al European Journal of Orthodontics 29 (2007) 471-476. Long term periodontal status of patient with mandibular lingual fixed retention.

[28] Artun and Urbye. The effect of orthodontic treatment on periodontal bone support in patient with advanced loss of marginal periodontium. American Journal of Orthodontics 93; 143-148; 1988

[29] Bantleon et al. Precise and time saving method of setting up an indirectly bonded retainer, American Journal of Orthodontics 93; 78-82, 1988

[30] Renkema et al, American Journal of Orthodontics and DentofacialOrthopedics. May 2011, vol 139, issue 5; 614-21.

[31] ChristosKatsaros et al, Unexpected complications of bonded mandibular lingual retainers. American journal of Orthodontics and DentofacialOrthopaedics. Dec 2007; 838-841. 